\title{
Immediate Effect of Intravitreal Bevacizumab Injection on Intraocular Pressure
}

\author{
Rabeeah Zafar ${ }^{1}$, Amna Rizwan ${ }^{2}$, Badar-ud-Din Ather Naeem ${ }^{3}$, Asfandyar Asghar ${ }^{4}$, Naila Obaid $^{5}$ \\ Department of Ophthalmology, ${ }^{1,2}$ Al-Shifa Trust Eye Hospital, ${ }^{3,4,5}$ Fauji Foundation Hospital, Rawalpindi
}

\begin{abstract}
Purpose: To determine the immediate effect of intravitreal Bevacizumab on intraocular pressure (IOP) in eyes with retinal vascular disorders.
\end{abstract}

Study Design: Interventional case series.

Place and Duration of Study: Ophthalmology Department, Fauji Foundation Hospital, Rawalpindi, from January 2019 to July 2019.

Methods: Patients of both genders between $15-80$ years of age suffering from retinal pathologies and suitable for intravitreal bevacizumab were included in the study. Systemic diseases like diabetes mellitus, hypertension and asthma were also considered in the data as an effect modifier for IOP change. IOP was measured before intravitreal injection (baseline) and at 5 and 30 minutes post-injection. Descriptive statistics were obtained using SPSS version 21.0 .

Results: One hundred and thirty-one eyes of 131 patients were included in the study out of which $23(18 \%)$ were males and $108(82.4 \%)$ were females. Mean age was $57.57 \pm 13.09$ years. Mean IOP at baseline was $16.16 \pm$ $2.52 \mathrm{~mm} \mathrm{Hg}$ which increased to a maximum of $44 \mathrm{~mm} \mathrm{Hg}$ at 5 minutes after injection in 108 eyes $(82.4 \%), \mathrm{p}=$ $0.005(\leq 0.05)$. At 30 minutes the IOP had fallen back to normal in 94 eyes $(71.7 \%), p=0.081$. IOP rise was not significantly correlated to gender, age, hypertension and asthma at any interval ( $p$ value $>0.05$ ). However, IOP rise was significantly correlated in diabetic patients at 30 minutes.

Conclusion: Significant IOP elevation has been observed after intravitreal bevacizumab in immediate postinjection period which warrants the monitoring of IOP in this critical period to avoid serious blinding complications.

Key Words: Bevacizumab, Vascular endothelial growth factor, Intraocular pressure, Goldmann applanation tonometer.

How to Cite this Article: Zafar R, Rizwan A, Naeem BDA, Asghar A, Obaid N. Immediate Effect of Intravitreal Bevacizumab Injection on Intraocular Pressure. Pak J Ophthalmol. 2020; 36 (4): 412-417.

Doi: https://doi.org/10.36351/pjo.v36i4.1109

\section{INTRODUCTION}

Retinal vascular disorders have been listed among the leading causes of irreversible blindness in developed

Correspondence: Rabeeah Zafar

Department of Ophthalmology, Al-Shifa Trust Eye Hospital, Rawalpindi

Email: rabeeah.zafar11@yahoo.com

Received: July 23, 2020

Accepted: September 3, 2020 as well as developing countries. ${ }^{1}$ Retinal hypoxia is the main initiating event resulting in release of vascular endothelial derived growth factor (VEGF) and pathological neovascularization with eventual catastrophic vascular leakage and anatomical disruption of retina, leading to visual deterioration. ${ }^{2}$ In the past, laser photocoagulation had been broadly utilized but the visual benefits were less encouraging. The advent of intravitreal anti-VEGF injections in early $21^{\text {st }}$ century led to a paradigm shift in the treatment of retinal vascular disorders owing to their efficacious profile in terms of visual improvement. ${ }^{3}$ 
Pegabtanib was the first anti-VEGF agent approved for intravitreal injection followed by ranibizumab and aflibercept with robust efficacy profile. ${ }^{4}$ They are in wide practice worldwide under different treatment regimens. ${ }^{5}$ Trials for newer anti-VEGFs are underway. ${ }^{6}$

Bevacizumab is a recombinant humanized full length monoclonal antibody which blocks angiogenesis by inhibiting VEGF isoforms. Its off label use has been widely carried out in ophthalmological practice since 2005; although it was initially devised for systemic treatment of metastatic colorectal cancer via intravenous route. ${ }^{7}$ The efficiency profile of bevacizumab is comparable to ranibizumab (Comparison of Age-related Macular Degeneration Treatments Trials research group) but its economic benefits are more being a cost effective substitute, hence it offers a better treatment option for a developing country like Pakistan. ${ }^{8}$ Despite an efficacious treatment modality, its ocular complications are very serious and can potentially result in vision loss. ${ }^{9,10}$ Of these, IOP elevation is the most frequently reported complication. This elevated IOP can affect vascular supply of optic nerve by elevating pressure gradient across lamina cribrosa and can damage it, resulting in irreversible blindness.

The rationale of our study was to demonstrate statistically conclusive IOP changes after intravitreal bevacizumab (IVB) injection. Moreover, to assess the outcome of influence of systemic disorders in terms of IOP changes after bevacizumab. Very inadequate local data is present in this regard. Hence, it would be a valuable source of information to compare statistics in Pakistani population and help ophthalmologists to modify the protocols regarding IOP monitoring after intravitreal bevacizumab. The objective of this study was to evaluate effect of intravitreal bevacizumab on IOP changes in immediate post-injection period.

\section{METHODS}

This interventional case series was conducted at Ophthalmology Department, Fauji Foundation Hospital, Rawalpindi from January, 2019 to July, 2019. Sample size was calculated using WHO calculator. A total of 131 patients were recruited from outdoor patient department using non probability consecutive sampling. Both genders were included between 15 - 80 years of age. Patients suffering from retinal pathologies and suitable for intravitreal bevacizumab were included. Systemic diseases like diabetes mellitus, hypertension and asthma were also considered in the data as an effect modifier for IOP change. Exclusion criteria consisted of any history of previous intravitreal injection, glaucoma, ocular trauma, and active ocular surface infections. Study was approved by institutional ethical review committee. Informed consent was taken from the patients.

Standard pre-injection ophthalmological examination was conducted. Baseline IOP was taken 5 minutes before giving injection using Goldmann Applanation Tonometer (AT 900 Haag-Streit) in the sitting position. Injection was given in minor operation theatre under standard sterile conditions. Topical proparacaine hydrochloride $0.5 \%$ was instilled thrice, 1 minute apart. It was followed by instilling 5\% povidone-iodine in cul-de-sac for 3 minutes. A dose of $1.25 \mathrm{mg} / 0.05 \mathrm{ml}$ bevacizumab (Avastin ${ }^{\circledR}$ by Roche Pakistan Ltd) was injected through pars plana according to phakic status of each eye by the same surgeon in all cases. After giving injection, IOP was recorded at 5 and 30 minutes in each eye. Normal IOP range was $11-21 \mathrm{~mm} \mathrm{Hg}$ and $22 \mathrm{~mm} \mathrm{Hg}$ and above was considered an IOP rise. Standard post-injection ophthalmic examination was done. All patients having IOP rise of $>30 \mathrm{~mm} \mathrm{Hg}$ after 30 minutes of injection were kept under observation with serial IOP monitoring and were dealt according to standard ophthalmic emergency protocol in order to lower IOP within safer range. Patients were prescribed topical ofloxacin $0.3 \%$ four times a day for 5 days. Data was recorded in a predesigned proforma.

Data analysis was done using Statistical Package for the Social Sciences (SPSS) version 21.0. Frequency and percentages were calculated for qualitative variables like gender and IOP rise. Mean and standard deviation were calculated for quantitative variables like age. Moreover, age, gender, diabetes mellitus, hypertension, asthma were controlled by stratification. To see correlation of IOP changes with these factors, chi-square test was applied with confidence interval of $95 \%$ and was considered significant if $p$ value was $\leq 0.05$.

\section{RESULTS}

Out of 131 patients (131 eyes) recruited in the study, 108 were females $(82 \%)$ whereas rest were males (18\%). Average age of the patients was $57.57 \pm 13.09$ years with minimum age being 20 years and maximum 
being 78 years. In 81 patients, injections were given in left eye $(61.8 \%)$ and in 50 patients in the right eye $(38.2 \%)$. Majority of the injected eyes had a diagnosis of diabetic retinopathy (as mentioned in Figure 1).

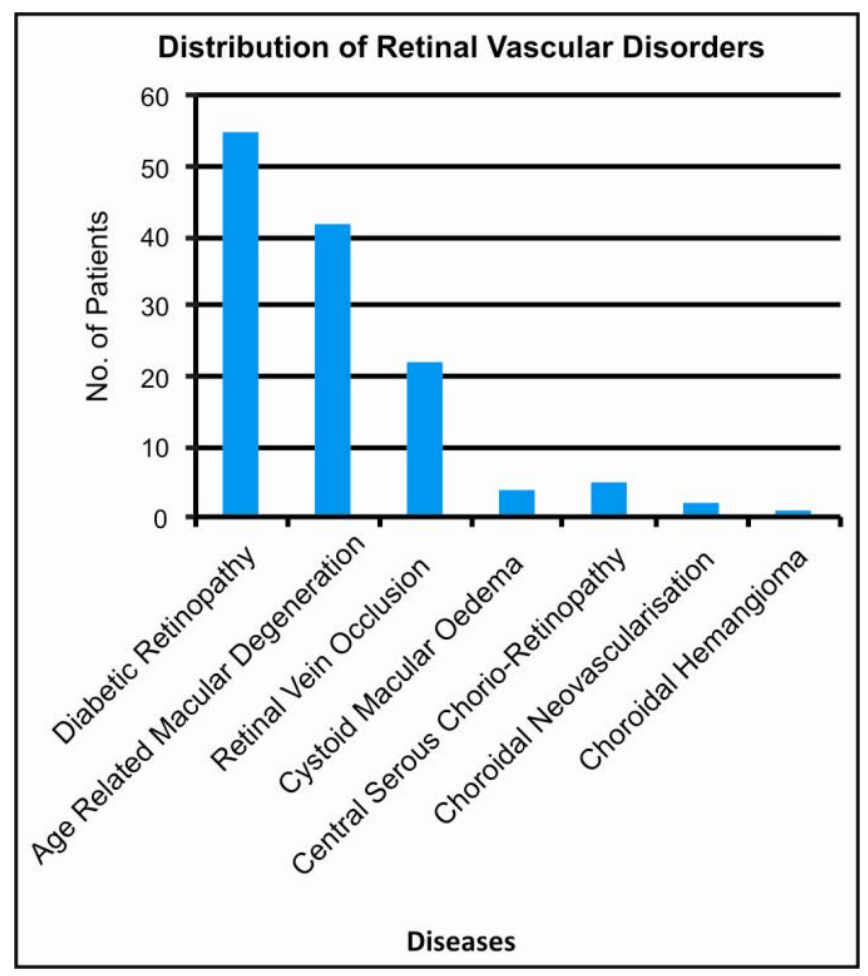

Fig. 1: Distribution of Retinal Disorders among Sample Population.

Mean IOPs and standard deviations for each tested variable are presented in Table 1. Mean IOP at baseline was $16.16 \pm 2.52 \mathrm{~mm} \mathrm{Hg}$, with maximum being $24 \mathrm{~mm} \mathrm{Hg}$. At 5 minutes post-injection, IOP rise $(\geq 22 \mathrm{~mm} \mathrm{Hg}$ ) was noted in 108 eyes $(82.4 \%)$. Maximum IOP recorded was $44 \mathrm{~mm} \mathrm{Hg}$, while mean IOP was $27.44 \pm 5.66 \mathrm{~mm} \mathrm{Hg}$. There was a statistically significant correlation between IOP at baseline and 5 minutes post-injection, applying chisquare test with $\mathrm{p}$ value $=0.005(\leq 0.05)$.

At 30 minutes post-injection, mean IOP was 19.97 $\pm 3.95 \mathrm{~mm} \mathrm{Hg}$ with maximum IOP recorded being 36 $\mathrm{mm} \mathrm{Hg}$. Statistically, an insignificant correlation was present between baseline IOP and IOP at 30 minutes with $\mathrm{p}$ value $=0.081(>0.05)$. (Please see Table 2$)$.

Diabetes mellitus was present in $86(65.6 \%)$ patients and IOP rise was noted in $69(63.9 \%)$ and 31 $(83.8 \%)$ eyes at 5 and 30 minutes, respectively. Statistically there was no difference in IOP at baseline and 5 minutes $(\mathrm{p}=0.251$ and 0.358$)$. However, at 30 minutes IOP had a statistically significant relation in diabetic patients i.e. $\mathrm{p}$ value $=0.006$.

Table 1: Comparison of IOP at baseline, 5 minutes and 30 minutes with Age, Gender, Diabetes, Hypertension, Asthma. $(I O P=$ intraocular pressure in millimeters of mercury $(\mathrm{mmHg}, \mathrm{N}=$ No. of patients).

\begin{tabular}{|c|c|c|c|c|c|}
\hline \multirow{7}{*}{ Age } & IOP & Groups & $\mathbf{N}$ & Mean & $\begin{array}{c}\text { Std. } \\
\text { Deviation }\end{array}$ \\
\hline & At & $15-50$ & 34 & 15.76 & 2.62 \\
\hline & baseline & $51-80$ & 97 & 16.30 & 2.48 \\
\hline & At 5 & $15-50$ & 34 & 26.38 & 4.68 \\
\hline & mins & $51-80$ & 97 & 27.80 & 5.94 \\
\hline & At 30 & $15-50$ & 34 & 19.03 & 2.44 \\
\hline & $\operatorname{mins}$ & $51-80$ & 97 & 20.30 & 4.32 \\
\hline \multirow{6}{*}{ Gender } & At & Male & 23 & 16.43 & 2.01 \\
\hline & baseline & Female & 108 & 16.10 & 2.61 \\
\hline & At 5 & Male & 23 & 27.57 & 5.48 \\
\hline & $\operatorname{mins}$ & Female & 108 & 27.41 & 5.72 \\
\hline & At 30 & Male & 23 & 20.35 & 4.37 \\
\hline & mins & Female & 108 & 19.89 & 3.88 \\
\hline \multirow{6}{*}{$\begin{array}{l}\text { Diabetes } \\
\text { Mellitus }\end{array}$} & At & Yes & 86 & 16.38 & 2.77 \\
\hline & baseline & No & 45 & 15.73 & 1.90 \\
\hline & At 5 & Yes & 86 & 27.84 & 6.19 \\
\hline & mins & No & 45 & 26.67 & 4.42 \\
\hline & At 30 & Yes & 86 & 20.56 & 4.38 \\
\hline & mins & No & 45 & 18.84 & 2.67 \\
\hline \multirow{6}{*}{ Hypertension } & At & Yes & 107 & 16.03 & 2.53 \\
\hline & baseline & No & 24 & 16.75 & 2.40 \\
\hline & At 5 & Yes & 107 & 27.55 & 5.77 \\
\hline & mins & No & 24 & 26.92 & 5.20 \\
\hline & At 30 & Yes & 107 & 19.85 & 4.10 \\
\hline & $\operatorname{mins}$ & No & 24 & 20.50 & 3.25 \\
\hline \multirow{6}{*}{ Asthma } & At & Yes & 8 & 16.88 & 0.641 \\
\hline & baseline & No & 123 & 16.11 & 2.59 \\
\hline & At 5 & Yes & 8 & 28.00 & 6.65 \\
\hline & $\operatorname{mins}$ & No & 123 & 27.40 & 5.62 \\
\hline & At 30 & Yes & 8 & 20.12 & 2.94 \\
\hline & mins & No & 123 & 19.96 & 4.02 \\
\hline
\end{tabular}

Among 131 patients, $107 \quad(81.7 \%)$ had hypertension. IOP at baseline, 5 minutes and 30 minutes post-injection was not significantly correlated among hypertensive patients $(\mathrm{p}=0.471,0.641$, and 0.540 respectively). Only $8(6.1 \%)$ patients in our data had asthma. IOP change was not significantly related among them ( $p$ value $>0.05$ ). Similarly, IOP changes among gender and age groups were not significantly related at baseline, 5 minutes and 30 minutes $(\mathrm{p}=$ $0.209,0.219,0.443$, and $\mathrm{p}=0.294,0.987,0.111$, respectively). Therefore, none of them were proven to be an effect modifiers regarding IOP rise based on chisquare test. 
Table 2: Stratification of IOP at Baseline with 5 Minutes and 30 Minutes.

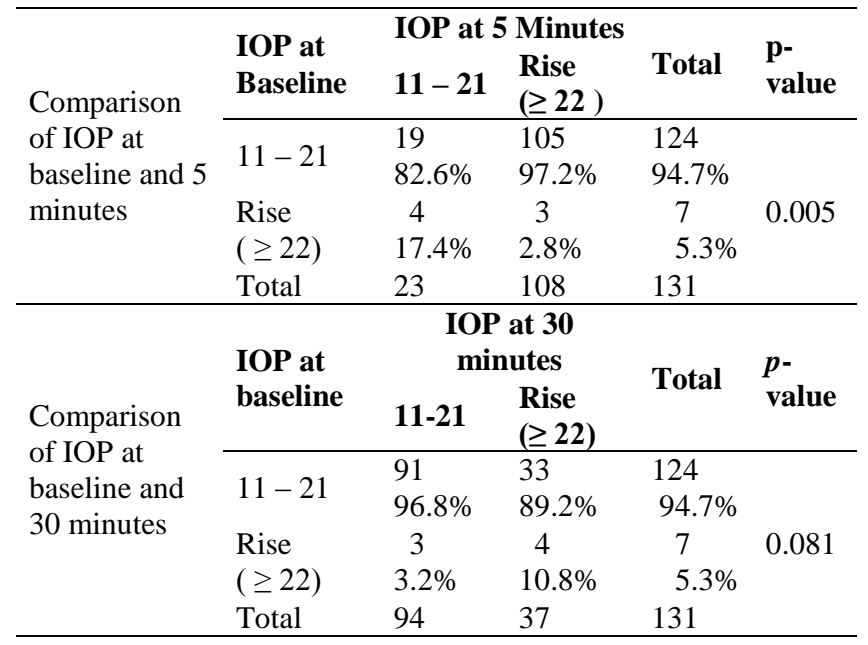

\section{DISCUSSION}

This study was conducted to investigate safety profile of bevacizumab based on severity of IOP rise in postinjection period. The authors were able to demonstrate that IOP rise was significantly high as early as 5 minutes post-intravitreal injection with maximum recording being $44 \mathrm{~mm} \mathrm{Hg}$. This high IOP potentially can result in sight-threatening event like central retinal vein occlusion. ${ }^{11}$ International literature well supports the observation of IOP spike after the drugs are introduced into the vitreous, be it anti-VEGFs, steroids or antibiotics. ${ }^{12,13}$ Among anti-VEGFs, bevacizumab has a tendency to raise IOP more as compared to other agents. ${ }^{14}$ Lemos-Reis et al. assessed IOP trends after intravitreal bevacizumab and reported very high IOPs of $>50 \mathrm{~mm} \mathrm{Hg}$ in $32 \%$ eyes. However, they did not reveal post-injection time at which such high IOP was recorded. ${ }^{15}$ Gismondi et al. observed IOP rise of $>30$ $\mathrm{mm} \mathrm{Hg}$ in $89 \%$ eyes as early as 5 seconds of intravitreal injection. ${ }^{16}$

Although exact mechanism of IOP rise after intravitreal injection is unknown. Some researchers have proposed that IOP elevation occurs due to temporary increase in vitreous volume, which is usually brief and no intervention like anterior chamber paracentesis is needed to lower IOP. ${ }^{17}$ However, Soheilian et al. suggested that intravitreal injections can result in significant IOP spikes and retinal nerve fiber loss in post-injection period. They had proven the efficacy of anterior chamber paracentesis in their randomized clinical study to lower this spike and hence, preventing the eventual nerve damage. ${ }^{18}$
Another possible mechanism of post-injection IOP spike could be multiple intravitreal injections. Falkenstein et al. supported this hypothesis by observing higher and prolonged IOP elevations in eyes with multiple previous injections. ${ }^{19}$ Moreover, multiple injections are also a risk factor for long term IOP elevation, and thus necessitating the need of early glaucoma surgery. ${ }^{20}$ Our study excluded patients with history of multiple previous injections and could not enlighten the effect of multiple injections on IOP trends.

Adverse events after intravitreal anti-VEGFs are under investigation since their revolutionary breakthrough and pricing advantages. Researchers all over the world are devising techniques to lower this expected IOP spike precluding ocular complications. Qureshi et al. studied IOP trends after intravitreal bevacizumab and also demonstrated the effect of preinjection ocular decompression at injection site on IOP rise by randomizing 100 eyes into 2 groups (IVB after decompression and IVB only). ${ }^{21}$ They observed a significant IOP rise in each study group. However, IOP rise in decompression group was less as compared to IOP in IVB only group and hence demonstrated the beneficial effects of this procedure in lowering the IOP spike in early post-injection period. ${ }^{21}$ Some researchers emphasize the need of antiglaucoma drugs before and after intravitreal injections. Some favour the protective role of post injection vitreous reflux in lowering IOP spikes. ${ }^{15}$ Kim et al. proposed that eyes having glaucoma took longer to lower the IOP after injection due to already compromised outflow tracts. ${ }^{22}$ Our study excluded glaucomatous eyes to avoid any confounding effect on IOP.

The strength of our study is the demonstration of effect of systemic diseases on IOP changes in postinjection period. The correlation of vascular disorders like diabetes and hypertension is well established with glaucoma. However, evidence of their role in IOP changes after intravitreal bevacizumab is lacking in non-glaucomatous population. In our study, diabetes has proven to have a statistically positive correlation with IOP rise at 30 minutes post injection $(\mathrm{p}<0.05)$.

In our study, despite the pressure rise in majority of eyes, IOP fell within a safer range $(<21 \mathrm{~mm} \mathrm{Hg})$ in two-third of the patients after 30 minutes of injection. These studies along with ours, gave an impression that IOP spike is must after intravitreal bevacizumab and is strongly related to the time at which IOP measurement is made, the greatest rise being observed shortly after 
injection. However, it is reassuring that IOP returned to normal or near-normal levels within 30 to 60 minutes postinjection.

The limitation of our study is that it was limited a single center. Further multicenter studies are required to assess the results in a larger population

\section{CONCLUSION}

This study positively contributes to clinical observation of significant IOP elevation after bevacizumab injection in immediate post-injection period. It may aid clinicians in improving and revising the protocols of serial IOP monitoring after intravitreal injections. The authors feel a strong need to further investigate the safe strategies to lower imminent IOP spikes. Moreover, the positive effect of diabetes mellitus on IOP elevation needs to be further investigated.

\section{Ethical Approval}

The study was approved by the Institutional review board/Ethical review board.

\section{Conflict of Interest}

Authors declared no conflict of interest.

\section{REFERENCES}

1. Quartilho A, Simkiss P, Zekite A, Xing W, Wormald $\mathbf{R}$, Bunce C. Leading causes of certifiable visual loss in England and Wales during the year ending 31 March 2013. Eye (Lond). 2016; 30 (4): 602-607.

Doi: 10.1038/eye.2015.288.

2. Bowling B. Kanski's Clinical Ophthalmology A Systemic Approach. 8th Ed. Edinburg: Elsevier; 2016: 521.

3. Amoaku WM, Chakravarthy U, Gale R, Gavin M, Ghanchi F, Gibson J, et al. Defining response to antiVEGF therapies in Neovascular AMD. Eye (Lond). 2015; 29: 721-731.

4. Nguyen CL, Oh LJ, Wong E, Wei J, Chiloy M. Antivascular endothelial growth factor for neovascular agerelated macular degeneration: a meta-analysis of randomized controlled trials. BMC Ophthalmol. 2018; 18: 130. https://doi.org/10.1186/s12886-018-0785-3.

5. Stewart MW. Treatment Strategies for Chorioretinal vascular Diseases: Advantages and Disadvantages of Individualised Therapy. EMJ Diabet. 2016; 4 (1): 9198.
6. Chexal S, Gunderson I, Berge B S, Jhaveri C. A Novel Compound for Treatment of Wet AMD. Retina Today; May/June, 2016: 72-75.

7. Ferrara N. From the discovery of vascular endothelial growth factor to the introduction of avastin in clinical trials - an interview with Napoleone Ferrara by Domenico Ribatti. Int J Dev Biol. 2011; 55 (4-5): 383388.

8. Van-Asten F, Michels CTJ, Hoyng CB, Van-der-wilt GJ, Klevering BJ, Rovers MM, et al. The costeffectiveness of bevacizumab, ranibizumab and aflibercept for the treatment of age-related macular degeneration-A cost-effectiveness analysis from a societal perspective. PLoS One, 2018; 13 (5): e0197670.

9. Nuzzi R, Tridico F. Local and systemic complications after intravitreal administration of anti-vascular endothelial growth factor agents in the treatment of different ocular diseases: a five-year retrospective study. Semin Ophthalmol. 2015; 30 (2): 129-135. Doi: $10.3109 / 08820538.2013 .835833$.

10. Xu Y, Tan CS. Safety and complications of intravitreal injections performed in an Asian population in Singapore. Int Ophthalmol. 2017; 37: 325-332.

11. Mansour AM, Bynoe LA, Welch JC, Pesavento R, Mahendradas P, Ziemssen $\mathbf{F}$, et al. Retinal vascular events after intravitreal bevacizumab. Acta Ophthalmol. 2010; 88 (7): 730-735.

12. Yuksel-Elgin $\mathbf{C}$, Elgin $\mathbf{C}$. Intraocular pressure elevation after intravitreal triamcinolone acetonide injection: a meta-analysis. Int J Ophthalmol. 2016; 9: 139-144.

13. Joseph A. Intravitreal injections: A brief note. Kerala J Ophthalmol. 2018; 30: 63-66.

14. Moraru A, Pinzaru G, Moțoc A, Costin D, Branişteanu D. Incidence of ocular hypertension after intravitreal injection of anti-VEGF agents in the treatment of neovascular AMD. Romanian J Ophthalmol. 2017; 61 (3): 207-211.

15. Lemos-Reis R, Moreira-Goncalves N, Melo AB, Carneiro AM, Falcao-Reis FM. Immediate effect of intravitreal injection of bevacizumab on intraocular pressure. Dove Press. Clin Ophthalmol. 2014; 8: 13831388.

16. Gismondi M, Salati C, Salvetat ML, Zeppieri M, Brusini P. Short-term effect of intravitreal injection of Ranibizumab (Lucentis) on intraocular pressure. J Glaucoma, 2009 Dec; 18 (9): 658-661. Doi: 10.1097/IJG.0b013e31819c4893.

17. Lee JW, Park H, Choi JH, Lee HJ, Moon SW, Kang JH, et al. Short-term changes of intraocular pressure and ocular perfusion pressure after intravitreal injection of bevacizumab or ranibizumab. BMC Ophthalmol. 2016; 16: 69. Doi: 10.1186/s12886-016-0255-8. 
18. Soheilian M, Karimi S, Montahae T, Nikkhah H, Mosavi SA. Effects of intravitreal injection of bevacizumab with or without anterior chamber paracentesis on intraocular pressure and peripapillary retinal nerve fiber layer thickness: a prospective study. Graefes Arch Clin Exp Ophthalmol. 2017; 255 (9): 1705-1712. Doi: 10.1007/s00417 017-3702-1.

19. Falkenstein IA, Cheng L, Freeman WR. Changes of intraocular pressure after intravitreal injection of bevacizumab (Avastin). Retina, 2007; 27: 1044-1047.

20. Eadie BD, Etminan M, Carleton BC, Maberley DA, Mikelberg FS. Association of Repeated Intravitreous Bevacizumab Injections with Risk for Glaucoma Surgery. JAMA Ophthalmol. 2017; 135 (4): 363-368. Doi: 10.1001/jamaophthalmol.2017.0059.

21. Qureshi NA, Mansoor H, Ahmed S, Zafar S, Asif M. Reducing intraocular-pressure spike after intravitrealbevacizumab injection with ocular decompression using a sterile cotton swab soaked in proparacaine $0.5 \%$ : A Quasi-Experimental study Taiwan J Ophthalmol. 2016; 6 (2): 75-78.

22. Kim JE, Mantravadi AV, Hur EY, Covert DJ. Shortterm Intraocular Pressure Changes Immediately After Intravitreal Injections of Anti-Vascular Endothelial Growth Factor Agents. Am J Ophthalmol. 2008; 146: 930-934.

\section{Authors' Designation and Contribution}

Rabeeah Zafar; Registrar: Concepts, Design, Data Acquisition, Data Analysis, Statistical Analysis, Manuscript Preparation.

Amna Rizwan; Registrar: Literature Research, Data Acquisition, Data Analysis.

Badar-ud-Din Ather Naeem; Professor: Concepts, Design, Manuscript editing, Manuscript review.

Asfandyar Asghar; Professor: Concepts, Design, Literature Research, Manuscript Editing, Manuscript Review.

Naila Obaid; Assistant Professor: Literature research, Data Acquisition, Manuscript Editing, Manuscript Review. 